\title{
Primary Neurilemmoma of the Thyroid Gland Clinically Mimicking Malignant Thyroid Nodule
}

\author{
Young Sub Lee · Jee Soon Kim · Arthur Minwoo Chung • Woo Chan Park ${ }^{1} \cdot$ Tae-Jung Kim \\ Departments of Hospital Pathology and ${ }^{1}$ Surgery, College of Medicine, The Catholic University of Korea, Seoul, Korea
}

Neurilemmomas, also known as schwannomas, are benign, slow-growing tumors that arise from Schwann cells of nerve roots. They can occur anywhere in the body, but those arising in the head and neck account for $25 \%$ to $45 \%$ of the cases. ${ }^{1}$ Primary neurilemmomas of the thyroid gland are very rare. Only 20 cases of primary thyroid neurilemmoma have been documented in the English literature. ${ }^{2}$ They were usually asymptomatic, and only two cases were found in children. ${ }^{3,4}$ Here we report this rare tumor in a 14-year-old girl, which was clinically mistaken for a malignant thyroid nodule.

\section{CASE REPORT}

A 14-year-old girl visited our hospital with a neck mass that had been present for 3 weeks without any symptoms. Ultrasonographic examination showed a lobulated, heterogeneous and hypoechoic nodule in the right thyroid lobe (Fig. 1A). Computed tomography with contrast showed a large hypodense mass in the right thyroid lobe with exophytic extension into the right strap muscle. The tumor was closely abutting and compressing the right tracheal wall (Fig. 1B). Fine needle aspiration cytology (FNAC) of the tumor showed a paucicellular smear composed of bland spindle-shaped cells in loosely cohesive aggregates (Fig. 1C). The patient underwent surgical resection. Intraoperative histological examination of a frozen section favored a benign mesenchymal tumor. Total thyroidectomy was performed; however,

\section{Corresponding Author}

Tae-Jung Kim, MD, PhD

Department of Pathology, Yeouido St. Mary's Hospital, 10 Yuksam-ro, Yeongdeungpogu, Seoul 07345, Korea

Tel: +82-2-3779-2157, Fax: +82-2-783-6648, E-mail: kimecho@catholic.ac.kr

Received: July 19, 2015 Revised: August 11, 2015

Accepted: August 26, 2015 because the lesion was severely adhered to the right tracheal wall, and the surgeon interpreted it as an invasion.

The entire thyroid gland was submitted for pathologic evaluation. On section, a well-demarcated, gray, firm mass was noted in the right thyroid lobe, measuring $3.0 \times 2.5 \times 2.0 \mathrm{~cm}$ (Fig. 1D). The tumor was completely surrounded by the capsule of the thyroid gland. Microscopic examination revealed a proliferation of uniformly spindled cells with a thick fibrous capsule (Fig. 2A). The tumor showed cellular areas with nuclear palisading (Antoni A) and pauci-cellular areas with myxoid change (Antoni B), a characteristic histologic feature of a neurilemmoma (Fig. 2B). There was extensive fibrosis around the tumor, but no perithyroidal tumor extension was found. Immunohistochemically, the tumor cells were positive for S-100 (Fig. 2C), Bcl-2, and fibroblast growth factor receptor 1 (FGFR1) (Fig. 2D), but negative for $\mathrm{CD} 34$ and platelet-derived growth factor receptor. Electron microscopy revealed complex intertwinings of slender cell processes outlined by a continuous basal lamina (Fig. 3). Publication of the case information and materials was approved by the Institutional Review Board of Yeouido St. Mary's Catholic Medical Center (SCMC IRB, Project Number SC12ZISE0225).

\section{DISCUSSION}

Non-epithelial thyroid tumors, including neurilemmomas, are very rare, accounting for less than $1 \%$ of all thyroid tumors. ${ }^{2}$ Delaney and Fry in $1964^{5}$ first reported a neurilemmoma arising in the thyroid gland. Only 20 cases of a primary thyroid neurilemmoma have been reported. ${ }^{2}$ All but two cases occurred in adults, ranging in age from 20 to 57 years. Baglaj et al. in $2004^{3}$ first described a primary neurilemmoma of the thyroid gland in a child. A 12-year-old girl underwent left thyroid lobectomy due 

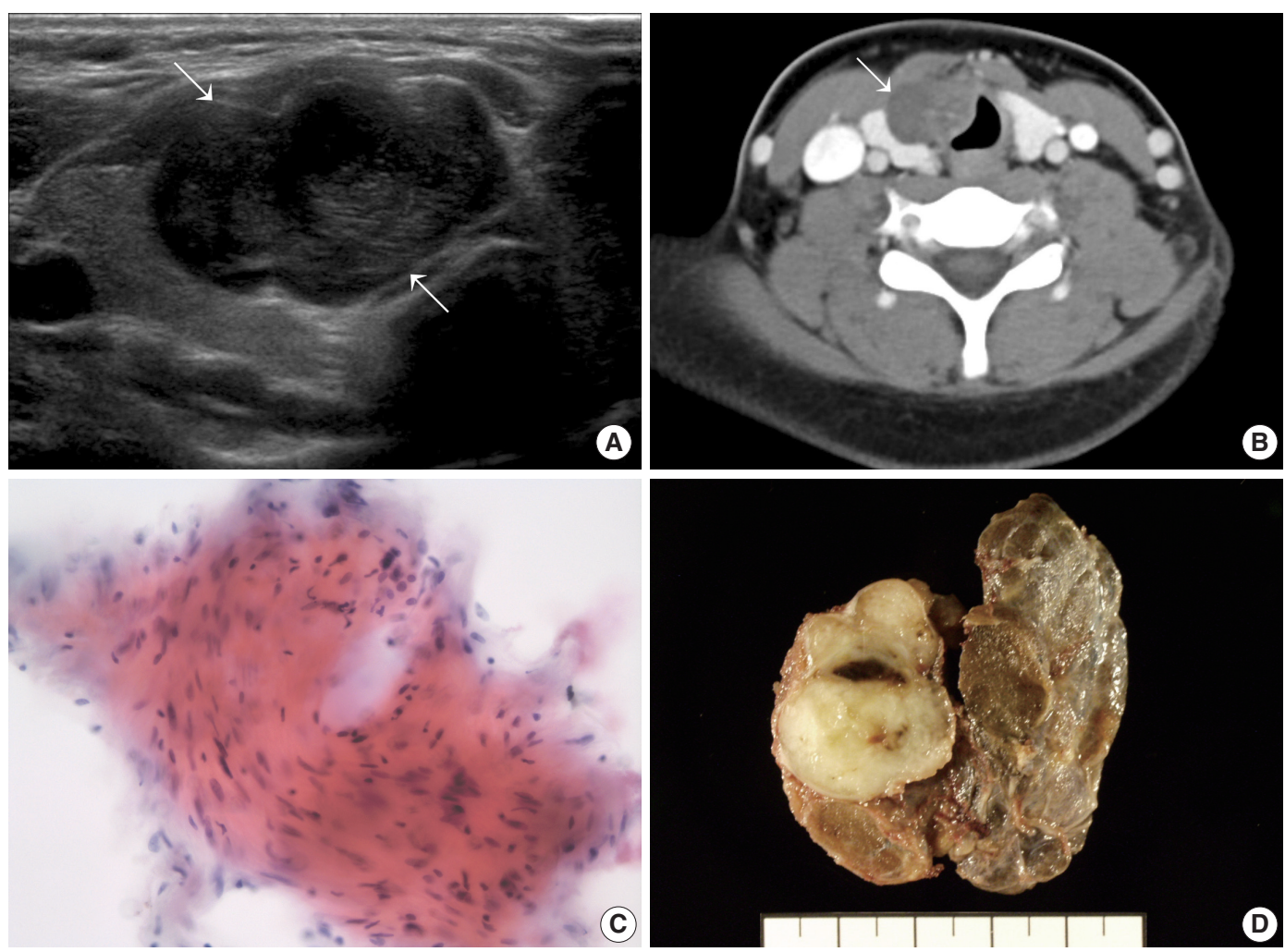

Fig. 1. Imaging, cytologic analysis, and gross examination of thyroidal mass. (A) Ultrasonography showing a lobulated, hypoechoic mass (arrow) in the subcapsular region of the right thyroid lobe. (B) Postcontrast computed tomography imaging revealing a hypodense right thyroidal mass (arrow) compressing the right tracheal wall. (C) Fine needle aspiration cytology with a loose cluster of bland-looking spindle cells. (D) The complete thyroidectomy specimen showing an encapsulated, gray, firm mass replacing the right upper lobe.

to an asymptomatic left lower neck swelling that had been present for 9 months. Ultrasonography showed a predominantly hypoechoic solid lesion measuring $1.7 \times 1.2 \times 2.6 \mathrm{~cm}$ within the left lobe. The second case of a primary thyroid neurilemmoma in a child was reported by An et al. in 2010. ${ }^{4}$ A 14-year-old male presented with an asymptomatic left intrathyroidal mass. Radiologic studies showed a hypoechoic left thyroid mass measuring $6.0 \times 4.5 \times 3.0 \mathrm{~cm}$ with cystic portions that was pushing the trachea toward the right. Both cases were diagnosed via postoperative pathological diagnosis as a neurilemmoma. The present case is the third occurrence of a primary thyroid neurilemmoma in a child.

Primary thyroid neurilemmomas should be differentiated from other benign spindle cell neoplasms of the thyroid glands, including vascular, smooth muscle, and fibroblastic tumors. ${ }^{6}$ The diagnosis can be made easily by identifying characteristic histologic features of neurilemmomas: alternating Antoni A and B areas with Verocay bodies (nuclear palisading around cell processes). ${ }^{6}$ Immunohistochemically, diffuse nuclear and cytoplasmic staining of S-100 protein can be helpful. Neurilemmomas aris- ing from the soft tissues of the neck and secondarily involving the thyroid gland should also be differentiated. ${ }^{7}$ Features suggesting the possibility of a primary thyroid origin may include a rim of thyroid tissue around the capsule and the tumor replacing a major portion of the thyroid gland.

The fibroblast growth factors and their receptors (FGFRs) are known to have a mitogenic effect on Schwann cells. ${ }^{8}$ In vestibular neurilemmomas, overexpression of FGFR1 is positively correlated with the incidence and growth rate of the tumor. ${ }^{9}$ Moreover, activation of FGFR1 may promote invasive behavior in vestibular neurilemmomas through ERK and Akt signaling pathways. ${ }^{10}$ In the present case, FGFR1 expression in tumor cells was identified by immunohistochemistry. Although there have been no studies of FGFR1 expression in primary thyroid neurilemmomas, it is suspected that increased expression of FGFR1 in tumor cells might play a role in the rapid growth of the tumor and its aggressive clinical behavior such as extensive tracheal adhesion.

In conclusion, it should be noted that primary neurilemmomas of the thyroid gland with marked adhesion can be clinical- 

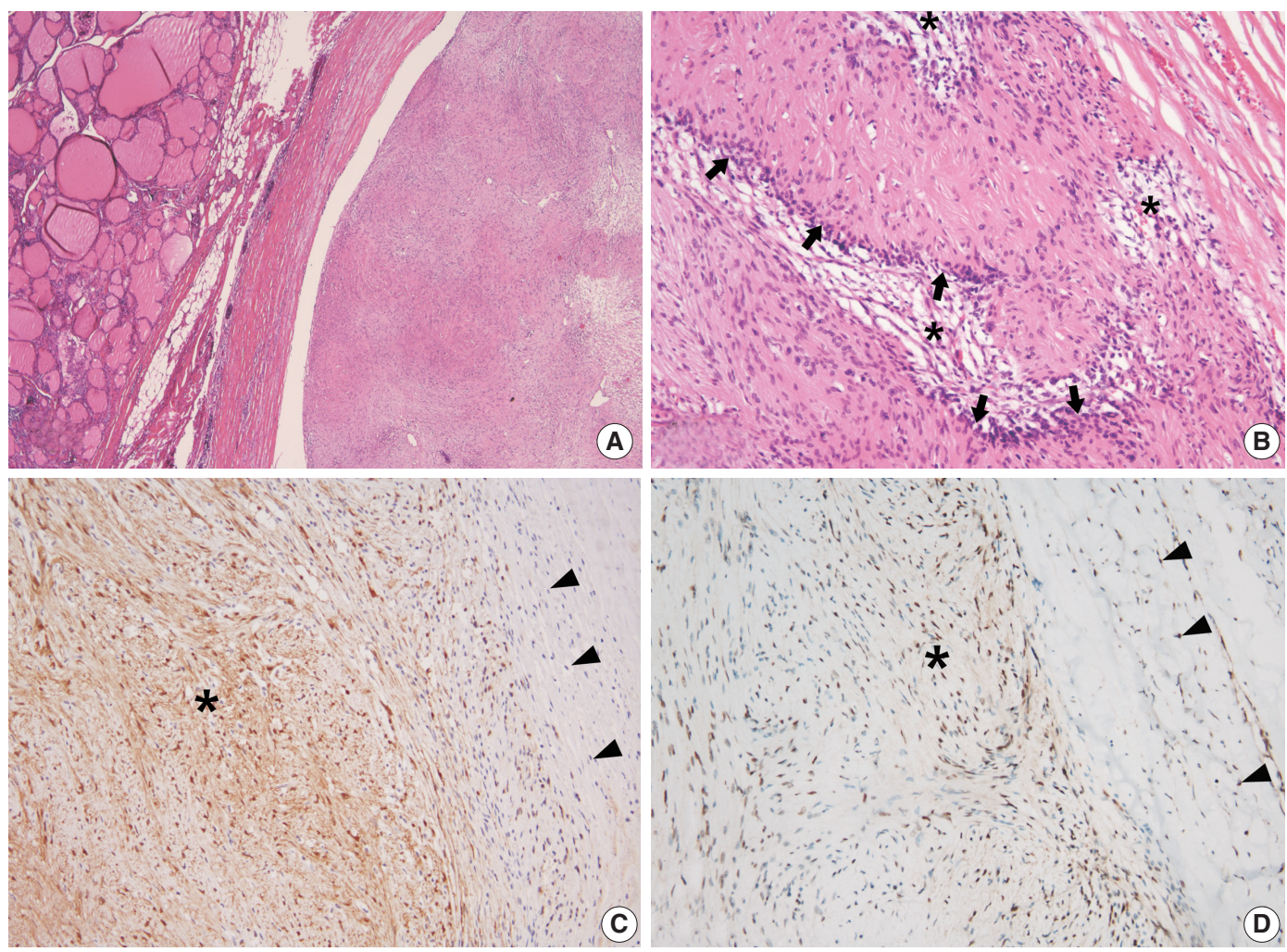

Fig. 2. Histologic and immunihistochemical analysis of the mass. (A) Low magnification view showing a spindle cell lesion (right) sharply demarcated from the adjacent normal thyroid tissue (left) by a thick fibrous capsule. (B) High magnification view revealing both cellular Antoni A areas (arrows) and loose paucicellular Antoni B areas (asterisks). (C) Tumor cells (asterisk) are positive and fibroblasts (arrowheads) are negative for S-100 protein on immunohistochemical staining. (D) Both tumor cells (asterisk) and fibroblasts (arrowheads) are positive for fibroblast growth factor receptor 1 on immunohistochemical staining.

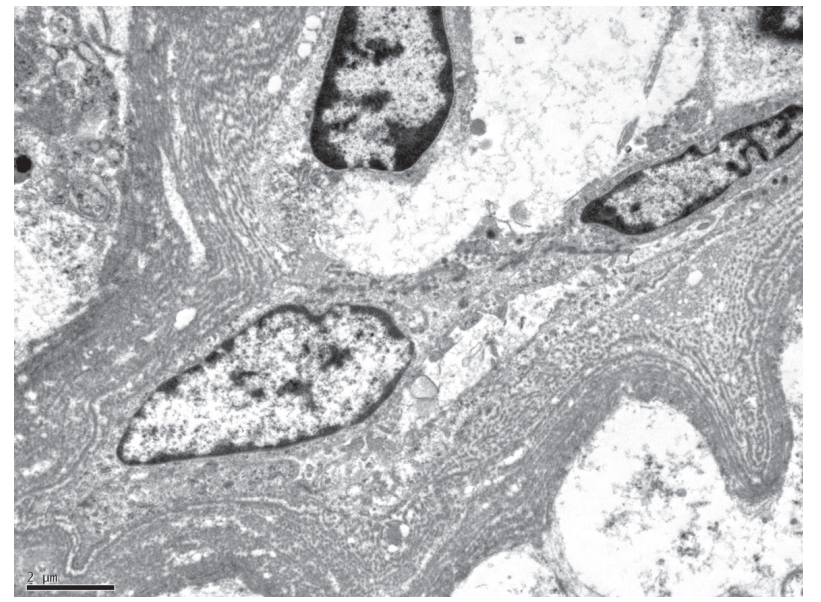

Fig. 3. Electron microscopy displaying long, enveloping cytoplasmic processes outlined by layers of discrete basal lamina.

ly misinterpreted as malignant thyroid nodules. Preoperative FNAC and intraoperative frozen section may help clinicians in making a proper decision on the surgical management of thyroid masses in children.

\section{Conflicts of Interest}

No potential conflict of interest relevant to this article was reported.

\section{REFERENCES}

1. Uri O, Baron E, Lefel O, Bitterman A. Primary schwannoma of the thyroid gland presenting as an asymptomatic cold nodule. Am J Otolaryngol 2009; 30: 427-9.

2. Graceffa G, Cipolla C, Florena AM, Gentile I, Pompei G, Latteri MA. Primary schwannoma of the thyroid gland involving the isthmus: report of a case. Surg Today 2013; 43: 106-9.

3. Baglaj M, Markowska-Woyciechowska A, Sawicz-Birkowska K, Dorobisz U. Primary neurilemmoma of the thyroid gland in a 12-year-old girl. J Pediatr Surg 2004; 39: 1418-20.

4. An J, Oh YL, Shin JH, Jeong HS. Primary schwannoma of the thyroid gland: a case report. Acta Cytol 2010; 54: 857-62.

5. Delaney WE, Fry KE. Neurilemoma of the thyroid gland. Ann Surg 1964; 160: 1014-7. 
6. Kandil E, Abdel Khalek M, Abdullah O, et al. Primary peripheral nerve sheath tumors of the thyroid gland. Thyroid 2010; 20: 583-6.

7. Badawi RA, Scott-Coombes D. Ancient schwannoma masquerading as a thyroid mass. Eur J Surg Oncol 2002; 28: 88-90.

8. Tzeng SF, Deibler GE, DeVries GH. Myelin basic protein and myelin basic protein peptides induce the proliferation of Schwann cells via ganglioside GM1 and the FGF receptor. Neurochem Res 1999; 24: $255-60$.
9. O'Reilly BF, Kishore A, Crowther JA, Smith C. Correlation of growth factor receptor expression with clinical growth in vestibular schwannomas. Otol Neurotol 2004; 25: 791-6.

10. Blair KJ, Kiang A, Wang-Rodriguez J, Yu MA, Doherty JK, Ongkeko WM. EGF and bFGF promote invasion that is modulated by PI3/ Akt kinase and Erk in vestibular schwannoma. Otol Neurotol 2011; 32: 308-14. 